“ (C) 2018 IEEE. Personal use of this material is permitted. Permission from IEEE must be obtained for all other uses, in any current or future media, including

reprinting/republishing this material for advertising or promotional purposes, creating new collective works, for resale or redistribution to servers or lists, or reuse of any copyrighted component of this work in other works." 


\title{
Narrowband Single-Pole Double-Throw Filtering Switch Based on Dielectric Resonator
}

\author{
Jin-Xu Xu ${ }^{\oplus}$, Student Member, IEEE, Xiu Yin Zhang ${ }^{\mathbb{1}}$, Senior Member, IEEE, \\ Hui-Yang Li, Student Member, IEEE, and Yang Yang ${ }^{\mathbb{1}}$, Senior Member, IEEE
}

\begin{abstract}
In this letter, a narrowband single-pole doublethrow (SPDT) filtering switch based on dielectric resonators (DRs) is presented. It consists of two DRs shared by two channels for size reduction. Printed circuit boards are embedded in the metal cavity to integrate the PIN diodes. The switching between two channels is enabled by controlling the PIN diodes connected to the two output feeding lines. The electromagnetic field distributions of the DR at the $\mathrm{TE}_{11 \delta}$ mode are studied to control the coupling between the DR and two output feeding lines. When one channel is on, the PIN diode for this channel is turned off, which does not introduce loss and affect the linearity. For the off-state channel, isolation is obtained by controlling the coupling between the DR and output feeding line, which is considerably enhanced. For demonstration, the DR filtering SPDT switch is implemented. The measured results exhibit that the proposed filtering SPDT switch has narrow bandwidth, low loss, high isolation, and high linearity.
\end{abstract}

Index Terms-Dielectric resonator (DR), filtering switch, high $Q$ factor, low loss, narrowband, single-pole double-throw (SPDT).

\section{INTRODUCTION}

$\mathbf{S}$ WITCHES and bandpass filters (BPFs) are key components in radio frequency front ends. Generally, they are cascaded, such as the single-pole double-throw (SPDT) switch and BPF in the time division duplex (TDD) subsystem in Fig. 1(a). To reduce the total loss that is the sum of the switch and BPF losses, co-designs of switch and BPF have been presented, with the topology shown in Fig. 1(b). The ON- and OFF-states are realized by embedding the PIN diodes or transistors into filter structures. For example, transistors can be utilized to switch ON and OFF two filters which are connected by $\mathrm{T}$ junction to realize the millimeter-wave filtering SPDT switches [1], [2]. In [3], the capacitively coupled $L C$ resonators with loaded PIN diodes are used to develop the filtering SPDT switch with compact size. Besides, the filtering SPDT switches have also been presented with compact size, low loss, or wide stopband performance in [4] and [5].

Manuscript received February 2, 2018; revised March 20, 2018; accepted April 30, 2018. Date of publication June 5, 2018; date of current version July 4, 2018. This work was supported by the National Natural Science Foundation of China under Grant 61725102 and Grant 61671210. (Corresponding author: Хiu Yin Zhang.)

J.-X. Xu is with the School of Electronic and Information Engineering, South China University of Technology, Guangzhou 510641, China, and also with the School of Electrical and Data Engineering, University of Technology Sydney, Ultimo, NSW 2007, Australia.

X. Y. Zhang and H.-Y. Li are with the School of Electronic and Information Engineering, South China University of Technology, Guangzhou 510641, China (e-mail: zhangxiuyin@ @otmail.com).

Y. Yang is with the School of Electrical and Data Engineering, University of Technology Sydney, Ultimo, NSW 2007, Australia.

Color versions of one or more of the figures in this paper are available online at http://ieeexplore.ieee.org.

Digital Object Identifier 10.1109/LMWC.2018.2837877

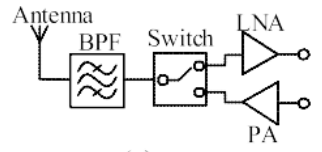

(a)

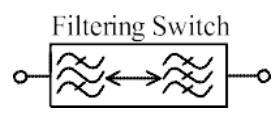

(b)
Fig. 1. (a) BPF and switch in TDD subsystem. (b) Filtering switch.

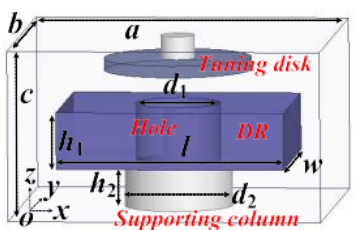

Fig. 2. Basic structure of the rectangular DR resonance cell.

Due to the $Q$ factor limitation, the above filtering switches [1]-[5] are not suitable for narrowband specifications, for example, less than $2 \%$ fractional bandwidth (FBW). Otherwise, the insertion loss would be very high. With the high $Q$ factor, dielectric resonators (DRs) are popularly employed in narrowband applications [6]-[8]. In [8], a filtering SPDT switch is implemented based on DRs. By embedding the PIN diodes into the nonresonant nodes (NRNs), the coupling between the DRs and NRNs can be controlled to enable the switching between two channels. However, three DRs are needed for the second-order filtering SPDT switch, occupying large circuit size.

In this letter, a new topology for the DR filtering SPDT switch is proposed. Two DRs are shared by two channels. Different from that in [8] by controlling the coupling between DRs and NRNs, the switching between two channels in this proposed design is enabled by controlling the coupling between output feeding lines and DR. As compared to [8], the proposed topology features simpler structure which can reduce one DR and two NRNs. Around 40\% reduction in size and weight is achieved. The cost is accordingly saved. The detailed design method is presented. The measured results show low loss of $0.5 \mathrm{~dB}$ with the FBW of $1.16 \%$, which is suitable for narrowband applications.

\section{TE $11 \delta$-MOde Dielectric RESONATOR}

In this design, the rectangular DR is utilized as a basic resonance cell, as shown in Fig. 2. It is composed of the metallic cavity with the tuning disk, DR, and supporting column inside. Different resonant modes coexist for such a structure. By properly controlling the height $\left(h_{1}\right)$, the length $(l)$, and the width $(W)$ of the DR, TE $11 \delta$ mode is the dominant resonant mode. A hole with a diameter of $d_{1}$ is dug at the center of the DR to separate the $\mathrm{TE}_{11 \delta}$ and $\mathrm{TM}_{11 \delta}$ modes.

For guiding the realization of the filtering switch, the electromagnetic (EM) fields of the rectangular DR are studied. 


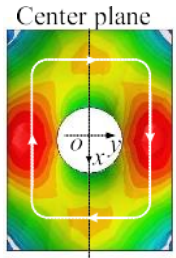

(a)

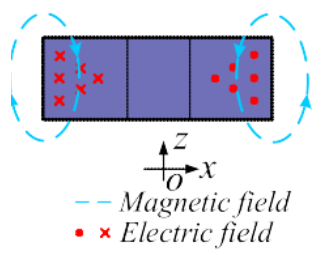

(b)
Fig. 3. (a) E-field of the DR in xoy plane. (b) EM field of the DR in xoz plane.

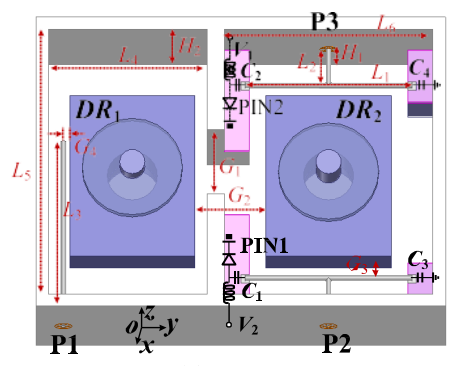

(a)

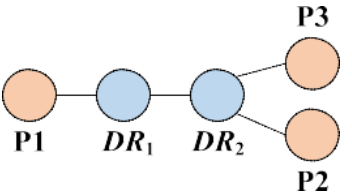

(b)
Fig. 4. DR filtering SPDT switch. (a) 3-D configuration. (b) Topology.

Initial parameters of the DR in Fig. 2 are chosen as follows (all in millimeter): $W=30, l=40, h_{1}=10, h_{2}=8, d_{1}=$ $12, d_{2}=19.5, a=66, b=38$, and $c=30$. Fig. 3(a) shows the magnitude and vector of the electric field ( $E$-field) of the $\mathrm{TE}_{11 \delta}$ mode DR in xoy plane, where red color represents the strongest density. It can be observed that the $E$-field is out of phase at two sides of the center plane. Based on the $E$-field distribution, the magnetic field ( $H$-field) distribution can be determined according to the right-hand screw rule. Fig. 3(b) shows the vector of the $H$-field seen from $x o z$ plane. It can be inferred that the $H$-field seen from xoy plane is symmetric with respect to the center plane.

\section{DR-BASED FILTERING SPDT SWITCH}

\section{A. Circuit Configuration}

Fig. 4(a) shows the 3-D configuration of the proposed SPDT filtering switch. It consists of a metallic cavity, two DRs $\left(\mathrm{DR}_{1}\right.$ and $\left.\mathrm{DR}_{2}\right)$, input feeding lines $(\mathrm{P} 1)$, and two output feeding lines (P2 and P3), where P2 and P3 are both coupled to $\mathrm{DR}_{2}$. One end of each output feeding line is connected to the switch circuitries including the PIN diodes (PIN1 or PIN2), inductors, and capacitors $\left(C_{1}\right.$ or $\left.C_{2}\right)$, while the other end connects to the capacitors $\left(C_{3}\right.$ or $\left.C_{4}\right)$. The topology is shown in Fig. 4(b). By controlling the PIN1 and PIN2, the circuit works in two states, namely, state 1 and state 2.

\section{B. Analysis of the DR-Based Filtering SPDT Switch}

When PIN1is turned ON and PIN2 is turned OFF, the filtering SPDT switch is in state 1; namely, signals at the operating band can be transmitted from P1 to P3 while P2 is isolated. The detailed analysis is addressed as follows.

In state 1, PIN1 is turned ON. One end of the output feeding line P2 that connects to the switch circuitry is approximate to short-circuited, because signals can pass through the switch circuitry ( $C_{1}$ and PIN1) to the ground. The other end of P2, which connects to $C_{3}$, is also equivalent to short-circuited when $C_{3}$ is chosen with a large capacitance. In this case, $\mathrm{P} 2$ can be regarded as symmetric structure with two short ends. The EM fields of the $\mathrm{DR}_{2}$ and $\mathrm{P} 2$ are illustrated in Fig. 5, which is similar to that in [8, Fig. 6]. As shown in Fig. 5(a),

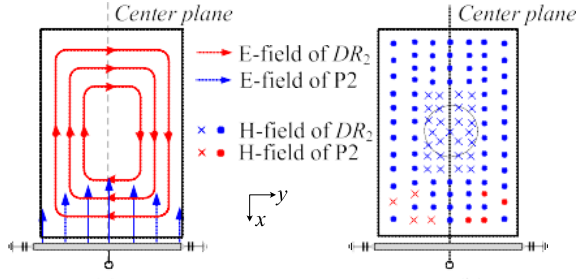

(a)

(b)

Fig. 5. EM-field distributions of $\mathrm{DR}_{2}$ and $\mathrm{P} 2$. (a) $E$-field. (b) $H$-field.

the $E$-field of $\mathrm{DR}_{2}$ at two sides of the center plane is out of phase while that of P2 is symmetric. It can be observed in Fig. 5(b) that the $H$ fields of $\mathrm{DR}_{2}$ and $\mathrm{P} 2$ are symmetric and out of phase, respectively. The electric and magnetic coupling coefficients $\left(k_{e}\right.$ and $k_{m}$ ) can be defined on the basis of the ratio of coupled energy to stored energy as

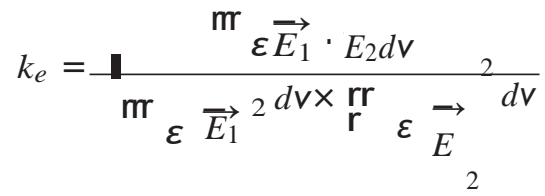

$$
\begin{aligned}
& k_{m}=\frac{\mathrm{mr} \vec{\mu} \cdot \vec{H} d v}{\mathrm{mr}_{\mu} \vec{H}_{1}^{2} d v \times{ }^{\mathrm{mr}} \mu \vec{H}_{2}^{2} d v}
\end{aligned}
$$

where $\vec{E}$ and $\vec{H}$ represent the electric and magnetic field vectors, and $v$ is the volume. From Fig. 5(a) and (b), both $k_{e}$ and $k_{m}$ between $\mathrm{DR}_{2}$ and $\mathrm{P} 2$ can be calculated as 0 based on (1) and (2), namely, $k_{e}=k_{m}=0$. Thus, the total coefficient $k=k_{e}+k_{m}$ is also 0. Accordingly, it can be concluded that the signals cannot be transmitted from P1 to P2 in state 1, resulting in isolated performance.

In state 1, PIN2 is turned OFF and, thus, P3 is not symmetric. Similar to the above analysis, we can know that the coupling coefficient between $\mathrm{DR}_{2}$ and $\mathrm{P} 3$ is nonzero, which can be tuned to meet the requirement of a BPF. Since the signals do not pass through PIN2, the circuit from P1 to P3 is the same as the conventional BPF. Hence, the BPF design method can be utilized. In this design, we adopt the Butterworth response. The lumped element values of the second-order prototype filter are selected to be: $g_{0}=1, g_{1}=1.4142, g_{2}=1.4142$, and $g_{3}=1$. The passband is required to be centered at $1.86 \mathrm{GHz}$ with a FBW of $1.15 \%$. The desired coupling coefficient $k$ and external quality factor $Q_{\mathrm{e}}$ can be calculated $Q_{\mathrm{e}}=130$ and $k=0.0081$. Consequently, the DR-based structure is constructed to meet these two values and good bandpass responses can be obtained.

In state 2, PIN1 is turned OFF and PIN2 is turned ON. Similar to the above analysis for state 1 , it can be known that P3 can be isolated from P1 and P2, while the circuit from $\mathrm{P} 1$ to $\mathrm{P} 2$ exhibits good bandpass responses. In conclusion, by turning ON or OFF PIN1 and PIN2, the signals at the operating passband can be transmitted from $\mathrm{P} 1$ to $\mathrm{P} 2$ or $\mathrm{P} 3$, featuring good performance of filtering SPDT switch.

\section{EXPERIMENT}

For demonstration, the SPDT DR filtering switch is implemented. Its 3-D view structure is shown in Fig. 4(a). The design parameters are given as follows (all in millimeter): $L_{1}=40, L_{2}=7.85, L_{3}=45, L_{4}=38, L_{5}=66, L_{6}=$ $50, G_{1}=8, G_{2}=16.5, G_{3}=3.85, G_{4}=2.5, H_{1}=13$, and $H_{2}=30$. The capacitances of $C_{1}-C_{4}$ are determined as: 


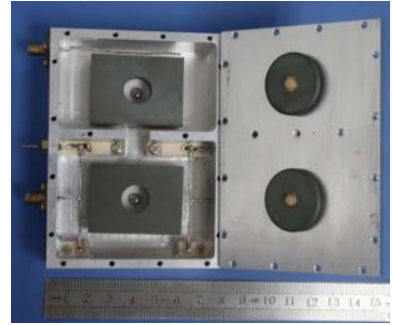

Fig. 6. Photograph of the fabricated DR-based filtering SPDT switch.

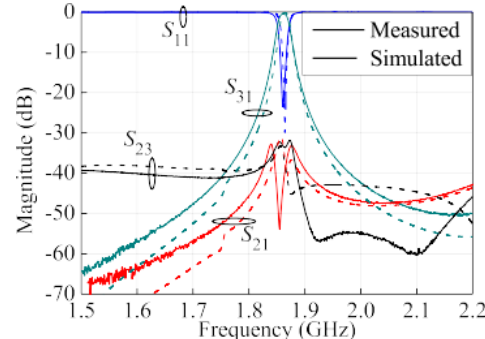

(a)

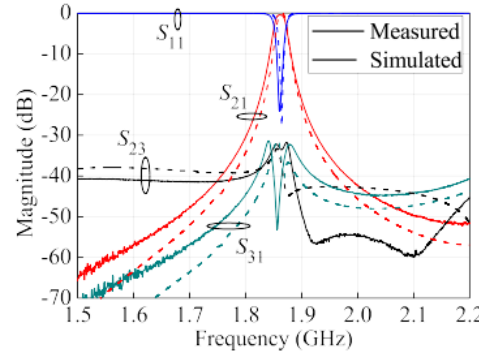

(b)

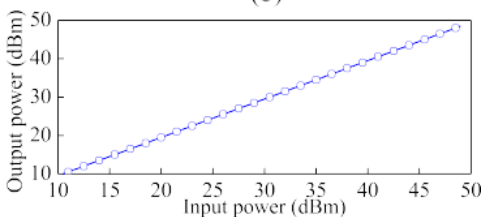

(c)

Fig. 7. Experimental results of the filtering SPDTswitch. (a) State 1. (b) State 2. (c) Measured output power versus input power of the oN-state channel.

$C_{1}=C_{2}=7 \mathrm{pF}$ and $C_{3}=C_{4}=15 \mathrm{pF}$. The dielectric material $\left(\varepsilon_{\mathrm{r}}=36.5\right.$ and $\left.\tan \sigma=0.00015\right)$ is from the Jiangsu Jiangjia Electronics Co., Ltd., Jiangsu, China. The diodes used in this design are implemented with Skyworks SMP 1302-085LF PIN diodes. The photograph of the fabricated filtering SPDT switch is shown in Fig. 6.

The measured results are obtained by using the Agilent E5071C network analyzer, which show good agreement with the simulated one, as shown in Fig. 7. In state 1, the measured $S_{31}$ is centered at $1.861 \mathrm{GHz}$ with a $3-\mathrm{dB}$ FBW of $1.16 \%$. The return loss is better than $20 \mathrm{~dB}$, and the insertion loss is $0.51 \mathrm{~dB}$ at the centered frequency, featuring good bandpass responses and low loss. Within a 15-dB return loss bandwidth, the difference between maximum and minimum insertion losses is small than $0.15 \mathrm{~dB}$. Due to the use of Butterworth filter prototype, there is no any transmission zero appeared. However, based on the design method in [8], transmission zeros are possible to be generated by introducing the NRN between $\mathrm{DR}_{1}$ and $\mathrm{DR}_{2}$ for better selectivity. The measured isolation between $\mathrm{P} 1$ and $\mathrm{P} 2$ is better than $40 \mathrm{~dB}$ at the center frequency and $32 \mathrm{~dB}$ within a frequency range from 1.5 to $2.2 \mathrm{GHz}$. The discrepancy between simulated and measured $S_{23}$ is found in Fig. 7(a) and (b). It is mainly due to
TABLE I

COMParison OF VARIOUS Filtering SPDT Switches

\begin{tabular}{r|c|c|c|c|c|c|c|c|c}
\hline Ref. & $\begin{array}{c}f \\
(\mathrm{GHz})\end{array}$ & N.R. & $\begin{array}{r}\text { Filter } \\
\text { order }\end{array}$ & $\begin{array}{c}\text { IL } \\
(\mathrm{dB})\end{array}$ & $\begin{array}{r}\text { FBW } \\
(\%)\end{array}$ & $\begin{array}{c}\text { ISO } \\
(\mathrm{dB}\end{array}$ & $\begin{array}{r}\mathrm{P}_{1 \mathrm{~dB}} \\
(\mathrm{dBm}\end{array}$ & Proces & Size $\left(\lambda_{\mathrm{g}}{ }^{2}\right)$ \\
\hline$[1]$ & 42 & 4 & 2 & 3.5 & 8 & 29 & 17.3 & $\mathrm{MMI}$ & N.A. \\
\hline$[4]$ & 1 & 5 & 3 & 0.97 & 10 & 40 & $>40$ & PCB & $0.16 \times 0.17$ \\
\hline$[8]$ & 1.831 & 3 & 2 & 0.4 & 1.3 & 45 & $>49$ & $\mathrm{DR}$ & $1.06 \times 0.46$ \\
\hline $\begin{array}{r}\text { This } \\
\text { work }\end{array}$ & 1.861 & 2 & 2 & 0.5 & 1.16 & 40 & $>49$ & $\mathrm{DR}$ & $0.61 \times 0.46$ \\
\hline
\end{tabular}

$\mathrm{N}$. R. means the number of resonators. IL and ISO denote the insertion loss and OFF-state isolation, respectively. N.A. means not available.

the fabrication errors of the length of the output feeding lines. In state 2 , the measured results are almost the same as those in state 1, as shown in Fig. 7(b), due to the symmetric circuit structure of $\mathrm{P} 2$ and $\mathrm{P} 3$. The measured $\mathrm{P}_{1 \mathrm{~dB}}$ of the ON-state channel is larger than $49 \mathrm{dBm}$, and the switch time is smaller than $1 \mu \mathrm{s}$.

Table I shows the comparison with some other filtering switches. In [1] and [4], the size of the filtering SPDT switches using monolithic microwave integrated circuit and printed circuit board is smaller than the proposed DR filtering SPDT switch. However, with the advantage of high $Q$ factor DR, our proposed design features lower loss, higher $\mathrm{P}_{1 \mathrm{~dB}}$, and narrower bandwidth. Compared to the second-order filtering SPDT switch using three DRs in [8], only two DRs are utilized in the proposed design, which simplifies the circuit structure. Moreover, around $40 \%$ reduction in size and weight is achieved.

\section{CONCLUSION}

This letter has presented a filtering switch using the rectangular DRs. Two DRs are shared by the two channels, resulting in size reduction. The EM-field distributions of the DR have been studied, and the detailed analysis of the filtering SPDT switch has been presented. A second-order DR-based filtering SPDT switch has been fabricated and measured. Low insertion loss, high isolation, and high-power handling capability are observed in the measured results. With the dual functions of filtering and switching, the proposed DR filtering SPDT switch is attractive in wireless systems.

\section{REFERENCES}

[1] S. F. Chao, "42 GHz MMIC SPDT bandpass filter-integrated switch using HEMT loaded coupled lines," Electron. Lett., vol. 48, no. 9, pp. 505-506, Apr. 2012.

[2] Z.-M. Tsai, Y.-S. Jiang, J. Lee, K.-Y. Lin, and H. Wang, "Analysis and design of bandpass single-pole-double-throw FET filter-integrated switches," IEEE Trans. Microw. Theory Techn., vol. 55, no. 8, pp. 1601-1610, Aug. 2007.

[3] J. Xu, Q.-H. Cai, Z.-Y. Chen, and Y.-Q. Du, "Quasi-lumped-element filter-integrated single-pole double-throw switch," IEEE Trans. Microw. Theory Techn., vol. 65, no. 11, pp. 4564-4571, Nov. 2017.

[4] C.-S. Chen, J.-F. Wu, and Y.-S. Lin, "Compact single-pole-double-throw switchable bandpass filter based on multicoupled line," IEEE Microw. Wireless Compon. Lett., vol. 24, no. 2, pp. 87-89, Feb. 2014.

[5] C.-S. Chen and Y.-S. Lin, "Single-pole double-throw switchable diplexer and single-pole quadruple-throw switchable quadplexer using capacitively loaded multicoupled line," IET Microw., Antennas Propag., vol. 9 , no. 14, pp. 1547-1557, Nov. 2015

[6] J.-X. Chen, Y. Zhan, W. Qin, Z.-H. Bao, and Q. Xue, "Analysis and design of balanced dielectric resonator bandpass filters," IEEE Trans. Microw. Theory Techn., vol. 64, no. 5, pp. 1476-1483, May 2016.

[7] S.-W. Wong, Z.-C. Zhang, S.-F. Feng, F.-C. Chen, L. Zhu, and Q.$\mathrm{X}$. Chu, "Triple-mode dielectric resonator diplexer for base-station applications," IEEE Trans. Microw. Theory Techn., vol. 63, no. 12, pp. 3947-3953, Dec. 2015.

[8] X. Y. Zhang, J.-X. Xu, and J.-X. Chen, "High-power filtering switch with low loss and high isolation based on dielectric resonator," IEEE Trans. Microw. Theory Techn., vol. 65, no. 6, pp. 2101-2110, Jun. 2017. 